\title{
A Method for Synthetic Aperture Compounding
}

\author{
Hansen, Jens Munk; Jensen, Jørgen Arendt
}

Published in:

Proceedings of IEEE International Ultrasonics Symposium

Link to article, DOI:

10.1109/ULTSYM.2010.0442

Publication date:

2010

Document Version

Early version, also known as pre-print

Link back to DTU Orbit

Citation (APA):

Hansen, J. M., \& Jensen, J. A. (2010). A Method for Synthetic Aperture Compounding. In Proceedings of IEEE International Ultrasonics Symposium (pp. 1743-1746). IEEE. https://doi.org/10.1109/ULTSYM.2010.0442

\section{General rights}

Copyright and moral rights for the publications made accessible in the public portal are retained by the authors and/or other copyright owners and it is a condition of accessing publications that users recognise and abide by the legal requirements associated with these rights.

- Users may download and print one copy of any publication from the public portal for the purpose of private study or research.

- You may not further distribute the material or use it for any profit-making activity or commercial gain

- You may freely distribute the URL identifying the publication in the public portal

If you believe that this document breaches copyright please contact us providing details, and we will remove access to the work immediately and investigate your claim. 


\title{
A Method for Synthetic Aperture Compounding
}

\author{
Jens Munk Hansen and Jørgen Arendt Jensen \\ Center for Fast Ultrasound Imaging, Dept. of Elec. Eng. Bldg. 349, \\ Technical University of Denmark, DK-2800 Kgs. Lyngby, Denmark
}

\begin{abstract}
An approach to perform ultrasound spatial compounding using synthetic aperture data is proposed. The approach allows compounding to be performed for any number of directions without reducing the frame rate or temporal resolution. It is demonstrated how the contrast is improved by compounding and the effect is quantized by speckle statistics and by computing contrast-to-noise ratios (CNR) from the resulting images. The method is validated using Field II simulations for a $7 \mathrm{MHz}, \lambda / 2$-pitch transducer with 192 elements with 64 elements active for each scan line. Circular regions (cysts) with a diameter of $5 \mathrm{~mm}$ and scattering levels ranging from -3 to $-12 \mathrm{~dB}$ relative to the background are imaged at 2 depths. Compound images composed of 1-5 images with an angular separation of 2 degrees are constructed and for the cysts at $-3,-6,-9$, and $-12 \mathrm{~dB}$, a CNR of $\mathbf{- 0 . 4 3 ,}-\mathbf{- 1 . 1 1}, \mathbf{- 1 . 4 4}$, and $\mathbf{- 1 . 9 1} \mathrm{dB}$ are obtained when using 5 images. Using the same RF data, a synthetic aperture image without compounding reveals a CNR of $-0.36,-0.93,-1.23$, and $-1.61 \mathrm{~dB}$ for the four cysts, respectively.
\end{abstract}

\section{INTRODUCTION}

Medical ultrasound imaging is used for many purposes, e.g. for localizing and classifying cysts, lesions, and other processes. Almost any mass is first observed using B-mode imaging and later classified using e.g. color flow, strain, or attenuation imaging. It is therefore important that the Bmode images have high contrast. Like all imaging modalities, ultrasound is subject to a number of inherent artifacts that compromise image quality. The most prominent artifact is the degradation by coherent wave interference, known as 'speckle', which gives a granular appearance to an otherwise homogeneous region of parenchyma [1], [2]. The speckle reduces image contrast and diminishes the possibilities for detection of low-contrast regions.

A successful approach to remedy the speckle artifacts is spatial compounding [3], [4], where images are acquired from a number of directions and combined after envelope-detection. Today, spatial compounding is implemented in all state of the art, high-end ultrasound systems and available when using a low pitch transducer with a fairly high number of independent channels [5]. Inherent in all compounding techniques is a loss in frame rate due to acquisition at multiple angles. This paper suggests an approach based on synthetic aperture imaging, where this can be avoided.

The organization of this paper is a follows: First, spatial compounding is described in more detail and some properties are introduced for measuring speckle statistics and contrast. Next, synthetic aperture imaging is explained and the proposal for using synthetic aperture data for compounding is presented.
In the results section, simulation results for a cyst phantom are presented together with speckle statistics and contrast-to-noise ratios for cysts with scattering levels, $\Delta V$ ranging from -3 to $-12 \mathrm{~dB}$ relative to the background. Finally, a discussion of the results is presented together with some future steps for further investigation of the proposed method.

\section{Methods}

\section{A. Compounding}

For conventional spatial compounding, a low-pitch linear array is used. A subset of the elements is selected as the current active aperture, and this aperture is used as a phased array to steer the beam in a direction making an angle $\theta$ with a normal to the transducer surface. The active aperture is then moved, until scan lines originating from the entire extent of the linear array are recorded. This procedure is repeated for angles $\theta_{i}, i=1, \ldots N_{\theta}$, where $N_{\theta}$ is the number of angles to be used for compounding. Next, the $N_{\theta}$ images are envelopedetected and added using scan-line conversion. An illustration of the scan line positions for $N_{\theta}=3$ is given in Fig. 1 . Note that the effective region of the image is reduced to the

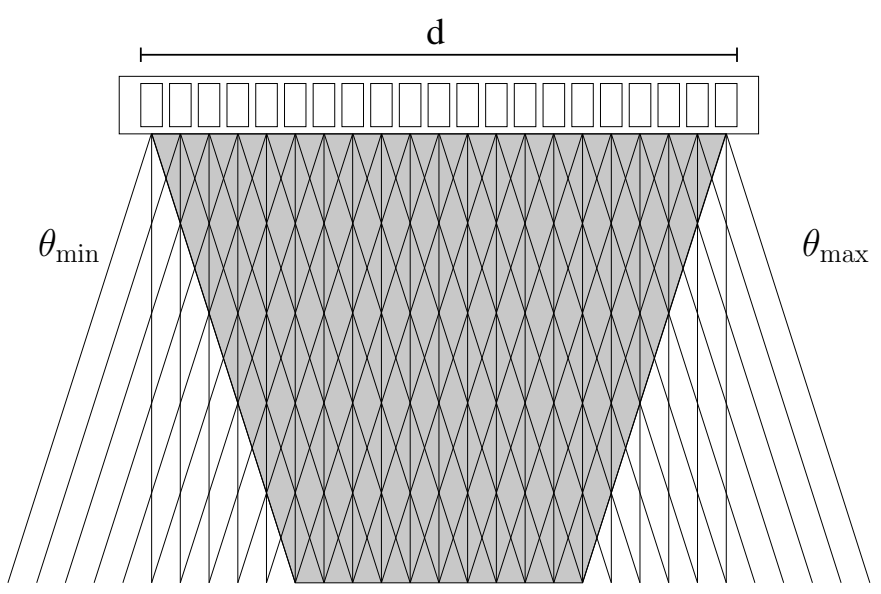

Fully compounded region

Figure 1: Illustration of scan line directions and origins used for spatial compounding. The example shows an image consisting of scan lines obtained using $N_{\theta}=3$ directions.

fully compounded image region determined by the length of the array $d$ and the beam angle extrema, $\theta_{\min }$ and $\theta_{\max }$. The resulting image has a reduced speckle appearance, a lower 
noise floor, boundaries subject to non-normal incidence are more visible, and image shadowing is confined to a smaller triangular region behind the attenuating masses or boundaries. For this study, only first order effects are considered, hence only the speckle pattern is analyzed.

To evaluate the image quality, one studies the magnitude $V$ of the received signal (found by envelope-detection). For first order statistics, the parameter of interest is the expectation value of $V, \mu_{V}=\mathbb{E}(V)$, in units of its standard deviation, $\sigma_{V}$. In the literature, this is referred to as the speckle signalto-noise ratio at a point,

$$
\mathrm{SNR}_{0}=\frac{\mu_{V}}{\sigma_{V}},
$$

and its inverse as the speckle contrast. The envelope-detected signals, $V$ follow Rayleigh statistics and for a fully developed speckle, the theoretical value of $\mathrm{SNR}_{0}$ is 1.91 [2], and if the $N_{\theta}$ images are uncorrelated, it is $1.91 \sqrt{N_{\theta}}$. Images used for compounding are not uncorrelated and therefore a smaller improvement of the signal-to-noise is obtained [6].

Another parameter of interest is the contrast-to-noise ratio for a region-of-interest (ROI) with a scattering level different from the background:

$$
\mathrm{CNR}=\frac{\mu_{V_{\mathrm{ROI}}}-\mu_{V_{\mathrm{B}}}}{\sqrt{\sigma_{V_{\mathrm{ROI}}}^{2}+\sigma_{V_{\mathrm{B}}}^{2}}},
$$

where $V_{\mathrm{ROI}}$ and $V_{\mathrm{B}}$ are the envelope-detected signals from the ROI and background, respectively.

A drawback of conventional compounding is either a reduction of the frame rate or a reduction of the true temporal resolution. In the case of the latter, the temporal resolution is reduced due to images being buffered to achieve an apparent high frame rate.

\section{B. Synthetic Aperture}

Synthetic aperture imaging can synthesize the transmit aperture [7], the receive aperture [8], or both [9]. A typical setup for doing both, is to acquire data by emitting with a single transducer element and receiving with all or a subset of the elements. This procedure is then repeated for all emissions and a complete data set is acquired. Using this data set, any beam and focusing can be synthesized.

For transmit focusing, delays and apodizations are calculated for each point in the image to construct signals, which at each point sum up coherently. For receive focusing, another set of delays and apodizations are applied to the signals received from the individual transducer elements and then a weighted sum is performed. In addition, the apodization can be adjusted to even out the resolution over a range of depths to maintain a constant resolution by realizing a fixed F-number using an expanding or contracting aperture for transmit and receive beamformation, respectively. A major advantage of synthetic aperture imaging is the possibility to focus everywhere in the image resulting in a better resolution. Further, this can be achieved using the same amount of time for data acquisition as for conventional imaging. The price for the resolution though, is a huge increase in RF data processing - an increase by the number of lines to beamform for each pulse emission.

\section{Synthetic Aperture Compounding}

To perform compounding using synthetic aperture data, apodizations are calculated for each image point corresponding to imaging the point using a focused beam centered around the point and making an angle $\theta$ with a normal to the transducer surface. In Fig. 2, it is shown for a single point, how the transmit apodization for an emission with transmit origin $\vec{r}_{\mathrm{xm}}$ are calculated using the orthogonal distance from the transmit origin to the "scan-line". Similarly, receive apodizations are

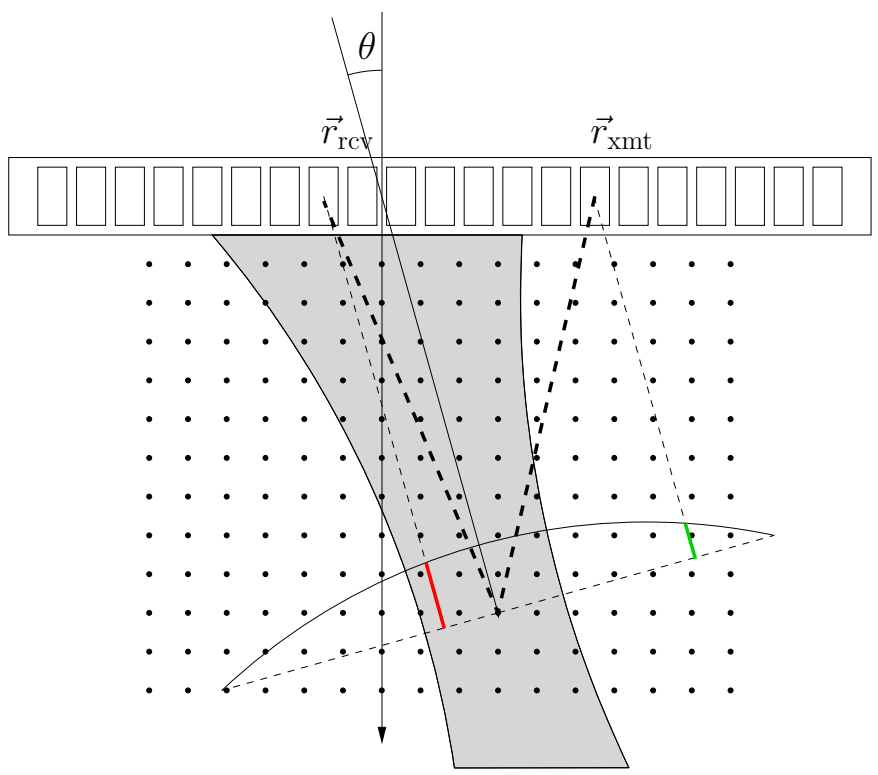

Figure 2: The example shows how the time-of-flight and apodizations are calculated for a single emission with transmit origin $\vec{r}_{\mathrm{xmt}}$ and a selected receive element positioned at $\vec{r}_{\mathrm{rcv}}$ for a single direction $\theta$. The length of the wave propagation path (thick dashed line) is used for calculating time-of-flight. The receive apodization is computed using the orthogonal distance from the receive element to the "scan line" and the value (red line) can be read-off from the arc representing an apodization function. Similarly, a transmit apodization (green line) is computed using the orthogonal distance from the origin of the transmission to the scan line. The grey area shows the "beam" that we are synthesizing by making the given apodization.

calculated for each point and applied to the signals received from the individual receiving elements of each transmission. The receive apodization values are likewise computed using the orthogonal distance from the "scan-line" to now the position of the receiving element. The delay calculation are identical to what is used for synthetic aperture imaging without compounding. The RF data are beamformed as in-phase and quadrature components, and the envelope computed as the absolute value. This rather complex procedure is repeated for angles $\theta_{i}, i=1, \ldots N_{\theta}$, where $N_{\theta}$ is the number of angles to be used for compounding. Finally, the compound image are constructed by addition or multiplication of the enveloped images. An implementation of this method uses a buffer for $N_{\theta}$ images and calculates the delays for each pixel only once. 


\begin{tabular}{lccc}
\hline \multicolumn{1}{c}{ \# of images } & 1 & 3 & 5 \\
\hline Compounding & 1.92 & 2.80 & 3.51 \\
SA Compounding & 1.87 & 1.92 & 2.00 \\
\hline
\end{tabular}

Further, the envelope can be computed for a pixel as soon as the RF data for the relevant emissions are processed to achieve the best possible temporal resolution.

\section{RESULTS}

\section{A. Simulation}

The proposed method for synthetic aperture compounding is implemented using a new software beamformation toolbox [10] and validated using Field II - a program for simulating ultrasound systems [11], [12]. A setup with a $7 \mathrm{MHz}, \lambda / 2$ pitch linear-array transducer with 192 elements are used for simulation. The emitted pulse is a single-cycle sinusoid and the temporal impulse response is modelled as a two-cycle sinusoid for both the transmitting and receiving aperture. The speckle pattern is modeled as the signal from a large collection of randomly placed scatterers with Gaussian amplitudes residing in a volume with dimensions (height-width-depth) $5 \times 20 \times 20$ $\mathrm{mm}$. Circular (cylindrical) regions with a diameter of $5 \mathrm{~mm}$ and scattering levels ranging from -3 to $-12 \mathrm{~dB}$ relative to the background are simulated at four positions by scaling the amplitudes accordingly. The random selection of point scatterers are chosen to have a density of at least 10 scatterers per resolution cell for any point in the image. The setup is shown in Fig. 3. Single-element emissions are simulated using

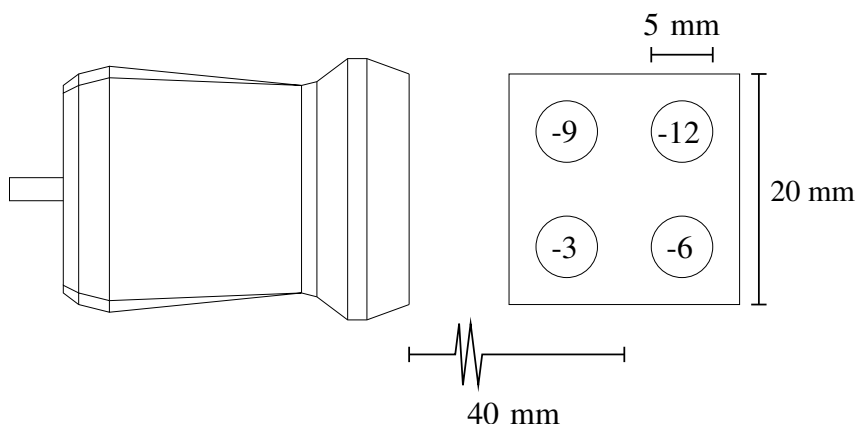

Figure 3: Simulation setup with a phased-array transducer and an image region of size (HWD) $5 \times 20 \times 20 \mathrm{~mm}$. containing 4 circular regions with a diameter of $5 \mathrm{~mm}$ and scattering levels ranging from -3 to $-12 \mathrm{~dB}$ relative to the background.

a sampling frequency of $120 \mathrm{MHz}$ and $\mathrm{RF}$ data are stored for all 192 elements, and all 192 elements are used for emission.

\section{B. Compounding}

For reference, a conventional compound image is created using 64 elements active for each scan line, dynamic receive focusing, and a Hamming apodization. Using random amplitudes for the scatter map, $\mathrm{SNR}_{0}$ values are computed for images using 1, 3, and 5 angles for compounding. The results are shown in Table I. The values increase when using an increasing number of angles for compounding.

In Fig. 4, images of the cyst phantom are constructed using 1,3 , and 5 angles for compounding. The CNR values for the
Table I: $\mathrm{SNR}_{0}$ for conv. and synthetic aperture compounding for images compounded of 1,3 , and 5 angles. The compounding is made using addition of the envelope-detected signals.

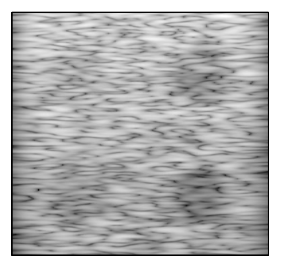

(a) 1 angle

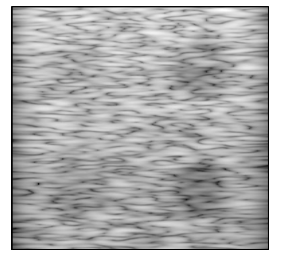

(d) 1 angle

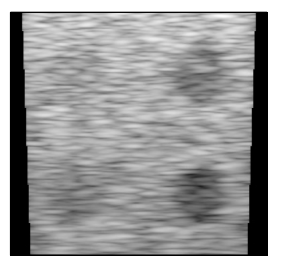

(b) 3 angles

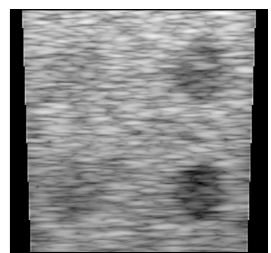

(e) 3 angles

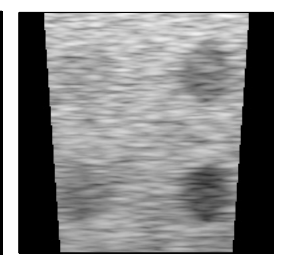

(c) 5 angles

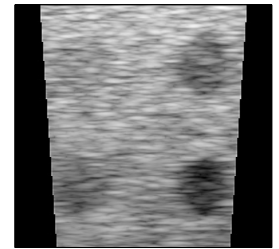

(f) 5 angles
Figure 4: Convention spatial compound images. The images (a) - (c) and (d) - (f) are compounded using addition and multiplication respectively. For all the images, only the fully compounded region is shown.

four cysts are computed in Table II. The values are increasing for an increasing number of images for all the cysts. It is worth noticing that the CNR is worse when compounding using multiplication.

\section{Synthetic Aperture Compounding}

Synthetic aperture compounding as described in Section II-C is applied to the simulated data using a Hamming window as the apodization function represented by the arc in Fig. 2. Following the same approach as for conventional compounding, $\mathrm{SNR}_{0}$ values are computed using random amplitudes for compounding using 1,3 , and 5 angles. The results are given in Table I. Note that the values are lower than the values obtained using conventional compounding. This can

\begin{tabular}{|c|c|c|c|c|c|c|c|}
\hline \multirow{2}{*}{\multicolumn{2}{|c|}{$\begin{array}{c}\text { Mode } \\
\text { \# of images }\end{array}$}} & \multicolumn{3}{|c|}{ Addition } & \multicolumn{3}{|c|}{ Multiplication } \\
\hline & & 1 & 3 & 5 & 1 & 3 & 5 \\
\hline \multirow{2}{*}{$\underline{\vartheta}$} & -3 & -0.48 & -0.73 & -0.83 & -0.48 & -0.67 & -0.78 \\
\hline & -6 & -0.67 & -1.21 & -1.65 & -0.67 & -1.15 & -1.51 \\
\hline \multirow{2}{*}{$\vec{\triangleleft}$} & -9 & -0.99 & -1.63 & -2.15 & -0.99 & -1.51 & -2.04 \\
\hline & -12 & -1.50 & -2.63 & -3.34 & -1.50 & -2.42 & -3.03 \\
\hline
\end{tabular}

Table II: Contrast-to-noise ratios for cysts at scattering levels, $\Delta V$, equal to $-3,-6,-9$, and -12 computed from conventional compound images constructed by addition respectively multiplication of 1,3 , and 5 images. 
be explained by the blurring lowering the variance of images obtained using conventional compounding.

In Fig. 5, synthetic aperture compound images of the cyst phantom are created using 1, 3, and 5 angles for compounding The CNR values for the four cysts are computed in Table III. The values are increasing for an increasing number of images for all the cysts. Again, it is worth noticing that the CNR is worse when compounding using multiplication.

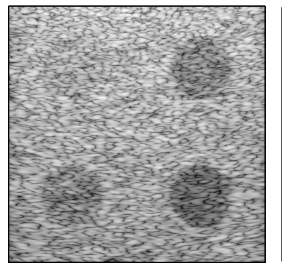

(a) 1 angle

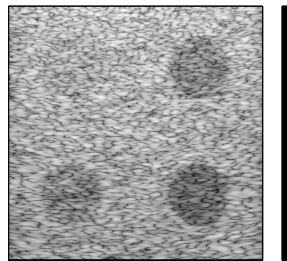

(d) 1 angle

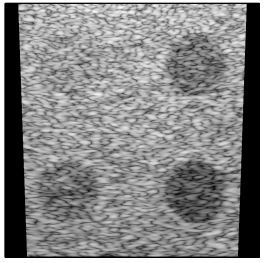

(b) 3 angles

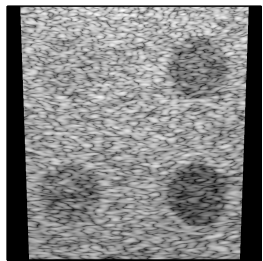

(e) 3 angles

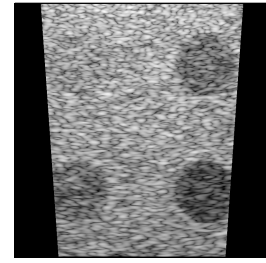

(c) 5 angles

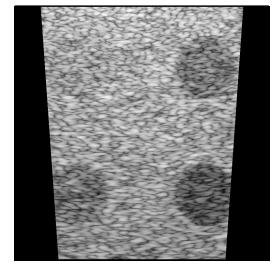

(f) 5 angles
Figure 5: Synthetic aperture compound images. The images (a) - (c) and (d) - (f) are compounded using addition and multiplication respectively. For all the images, only the fully compounded region is shown.

\begin{tabular}{|c|c|c|c|c|c|c|c|}
\hline \multirow{2}{*}{\multicolumn{2}{|c|}{$\begin{array}{c}\text { Mode } \\
\text { \# of images }\end{array}$}} & \multicolumn{3}{|c|}{ Addition } & \multicolumn{3}{|c|}{ Multiplication } \\
\hline & & 1 & 3 & 5 & 1 & 3 & 5 \\
\hline \multirow{4}{*}{$\begin{array}{l}\bar{\theta} \\
\vec{\theta} \\
\vec{y}\end{array}$} & -3 & -0.36 & -0.40 & -0.43 & -0.36 & -0.38 & -0.40 \\
\hline & -6 & -0.93 & -1.02 & -1.11 & -0.93 & -0.98 & -1.05 \\
\hline & -9 & -1.23 & -1.34 & -1.44 & -1.23 & -1.29 & -1.36 \\
\hline & -12 & -1.61 & -1.76 & -1.91 & -1.61 & -1.69 & -1.80 \\
\hline
\end{tabular}

Table III: Contrast-to-noise ratios for cysts at scattering levels, $\Delta V$, equal to $-3,-6,-9$, and -12 computed from synthetic aperture compound images constructed by addition respectively multiplication of 1,3 , and 5 images.

\section{CONCLUSION}

For all cysts imaged using spatial compounding, an improved speckle signal-to-noise ratio is obtained, when increasing the number of images and this in agreement with the results of [6]. As a consequence, the contrast is improved and low-contrast regions are easier to detect. Using synthetic aperture, an increased resolution is obtained, which is evident from Fig.5. The fact that using the proposal for synthetic aperture compounding, the speckle is reduced and the contrast improved is evident from the results of Table I and Table III and this opens for further applications of synthetic aperture imaging worth investigating. In particularly, better contrast can easily be obtained by trading off resolution by using a smaller transmit or receive aperture. For an implementation, the obvious choice is to use fewer emissions to also obtain a higher temporal resolution.

Next steps are to collect synthetic aperture data with our new research scanner SARUS [13], validate the method on invivo data, investigate the resolution trade-off when using fewer transmissions, and finally to implement synthetic aperture compounding for real-time in-vivo imaging to validate the method using pre-clinical trials.

\section{REFERENCES}

[1] C. Burckhardt, "Speckle in ultrasound b-mode scans," IEEE Trans. Son Ultrason., vol. SU-25, no. 1, pp. 1-6, Jan. 1978.

[2] R. F. Wagner, S. W. Smith, J. M. Sandrick, and H. Lopez, "Statistics of speckle in ultrasound B-scans," IEEE Trans. Son. Ultrason., vol. 30, pp. 156-163, 1983.

[3] D. Shattuck and O. von Ramm, "Compounding scanning with a phased array," Ultrason. Imaging, vol. 4, pp. 93-107, 1982.

[4] G. E. Trahey, S. W. Smith, and O. T. von Ramm, "Speckle pattern correlation with lateral aperture translation: Experimental results and implications for spatial compounding," IEEE Trans. Ultrason., Ferroelec., Freq. Contr., vol. UFFC-33, pp. 257-264, 1986.

[5] R. Entrekin, P. Jackson, and B. Porter, "Real time spatial compound imaging in breast ultrasound: technology and early clinical experience," medicamundi, vol. 43, pp. 35-43, September 1999.

[6] S. K. Jespersen, J. E. Wilhjelm, and H. Sillesen, "Multi-angle compound imaging," Ultrason. Imaging, vol. 20, pp. 81-102, 1998.

[7] C. R. Hazard and G. R. Lockwood, "Theoretical assessment of a synthetic aperture beamformer for real-time 3-D imaging," IEEE Trans. Ultrason., Ferroelec., Freq. Contr., vol. 46, pp. 972-980, 1999.

[8] L. F. Nock and G. E. Trahey, "Synthetic aperture imaging in medical ultrasound with correction for motion artifacts," in Proc. IEEE Ultrason. Symp., 1990, pp. 1597-1601.

[9] G. R. Lockwood, J. R. Talman, and S. S. Brunke, "Real-time 3-D ultrasound imaging using sparse synthetic aperture beamforming," IEEE Trans. Ultrason., Ferroelec., Freq. Contr., vol. 45, pp. 980-988, 1998.

[10] J. M. Hansen and J. A. Jensen, "An object-oriented multi-threaded software beam formation toolbox," in Proc. SPIE - Medical Imaging - Ultrasonic Imaging and Signal Processsing, 2011, submitted.

[11] J. A. Jensen and N. B. Svendsen, "Calculation of Pressure Fields from Arbitrarily Shaped, Apodized, and Excited Ultrasound Transducers," IEEE Trans. Ultrason., Ferroelec., Freq. Contr., vol. 39, pp. 262-267, 1992.

[12] J. A. Jensen, "Field: A Program for Simulating Ultrasound Systems," Med. Biol. Eng. Comp., vol. 10th Nordic-Baltic Conference on Biomedical Imaging, Vol. 4, Supplement 1, Part 1, pp. 351-353, 1996 b.

[13] J. A. Jensen, M. Hansen, B. G. Tomov, S. I. Nikolov, and H. HoltenLund, "System architecture of an experimental synthetic aperture real time ultrasound system," in Proc. IEEE Ultrason. Symp., Oct. 2007, pp. 636-640. 